\title{
Myeloid Engraftment
}

National Cancer Institute

\section{Source}

National Cancer Institute. Myeloid Engraftment. NCI Thesaurus. Code C147533.

Myeloid recovery after bone marrow transplantation. It manifests with the production of new blood cells. It takes approximately two to four weeks after the bone marrow is infused with the transplanted stem cells for the recovery to occur. 